\title{
MODULUS OF CONTINUITY OF $p$-DIRICHLET SOLUTIONS IN A METRIC MEASURE SPACE
}

\author{
Tsubasa Itoh \\ Hokkaido University, Department of Mathematics \\ Sapporo 060-0810, Japan; tsubasa@math.sci.hokudai.ac.jp
}

\begin{abstract}
Let $1<p<\infty$ and let $X$ be a metric measure space with a doubling measure and a $(1, p)$-Poincaré inequality. Let $\Omega$ be a bounded domain in $X$. For a function $f$ on $\partial \Omega$ we denote by $\mathcal{P}_{\Omega} f$ the $p$-Dirichlet solution of $f$ over $\Omega$. It is well known that if $\Omega$ is $p$-regular and $f \in C(\partial \Omega)$, then $\mathcal{P}_{\Omega} f$ is $p$-harmonic in $\Omega$ and continuous in $\bar{\Omega}$. We characterize the family of domains $\Omega$ such that improved continuity of boundary functions $f$ ensures improved continuity of $\mathcal{P}_{\Omega} f$. We specify such improved continuity if $X$ is Ahlfors regular and $X \backslash \Omega$ is uniformly $p$-fat.
\end{abstract}

\section{Introduction}

Let $X=(X, d, \mu)$ be a complete connected metric measure space endowed with a metric $d$ and a positive complete Borel measure $\mu$ such that $0<\mu(U)<\infty$ for all non-empty bounded open sets $U$.

By the symbol $C$ we denote an absolute positive constant whose value is unimportant and may change from line to line. Let $B(x, r)=\{y \in X: d(x, y)<r\}$ denote the open ball centered at $x$ with radius $r$. We assume that $\mu$ is doubling, i.e., there is a constant $C \geq 1$ such that $\mu(B(x, 2 r)) \leq C \mu(B(x, r))$ for every $x \in X$ and $r>0$. Let $1<p<\infty$. We assume that $X$ admits a $(1, p)$-Poincaré inequality.

We denote by $\operatorname{Cap}_{p}$ the $p$-capacity defined on $X$ (Definition 2.5). Let $\Omega \subset X$ be a bounded domain with $\operatorname{Cap}_{p}(X \backslash \Omega)>0$. For a function $f$ on $\partial \Omega$ we donate by $\mathcal{P}_{\Omega} f$ the $p$-Perron solution of $f$ over $\Omega$. A point $\xi \in \partial \Omega$ is said to be a p-regular point (with respect to the $p$-Dirichlet problem) if

$$
\lim _{\Omega \ni x \rightarrow \xi} \mathcal{P}_{\Omega} f(x)=f(\xi)
$$

for every $f \in C(\partial \Omega)$. If every boundary point is a $p$-regular point, then $\Omega$ is called $p$ regular. It is well known that if $\Omega$ is $p$-regular and $f \in C(\partial \Omega)$, then $\mathcal{P}_{\Omega} f$ is $p$-harmonic in $\Omega$ and continuous in $\bar{\Omega}$. It is natural to raise the following question:

Question 1.1. Does improved continuity of a boundary function $f$ guarantee improved continuity of $\mathcal{P}_{\Omega} f$ ?

Aikawa and Shanmugalingam [3] studied this question in the context of Hölder continuity. Aikawa [2] investigated this question in the context of general modulus of continuity for the classical setting, i.e., for harmonic functions in a Euclidean domain.

doi:10.5186/aasfm.2012.3741

2010 Mathematics Subject Classification: Primary 31E05, 31C45, 35J60.

Key words: Modulus of continuity, $p$-harmonic, $p$-Dirichlet solution, Metric measure space, $p$-capacity.

Research Fellow of the Japan Society for the Promotion of Science. 
The purpose of this paper is to study this question in the context of general modulus of continuity in a metric measure space.

Let $\mathcal{M}$ be the family of all positive nondecreasing concave functions $\psi$ on $(0, \infty)$ with $\psi(0)=\lim _{t \rightarrow 0} \psi(t)=0$. We say that $f$ is $\psi$-Hölder continuous if $|f(x)-f(y)| \leq$ $C \psi(d(x, y))$. The modulus of continuity of a uniformly continuous function on any geodesic space is comparable to a certain concave function. See [5, Chapter $2 \S 6]$ and Propositions 2.13 and 2.14. The author would like to thank Kuroda for drawing his attention to [5]. Therefore, we have only to check $\psi$-Hölder continuity for $\psi \in \mathcal{M}$ to study Question 1.1 in the context of modulus of continuity.

As a typical example of $\psi \in \mathcal{M}$ we consider $\psi_{\alpha \beta}$ defined by

$$
\psi_{\alpha \beta}(t)= \begin{cases}t^{\alpha}(-\log t)^{-\beta} & \text { for } 0<t<t_{0}, \\ t_{0}^{\alpha}\left(-\log t_{0}\right)^{-\beta} & \text { for } t \geq t_{0} .\end{cases}
$$

where either $0<\alpha<1$ and $\beta \in \mathbf{R}$ or $\alpha=0$ and $\beta>0$; and $t_{0}$ is so small that $\psi_{\alpha \beta} \in \mathcal{M}$. In particular, we write $\varphi_{\alpha}=\psi_{\alpha 0}$, and we say that $f$ is $\alpha$-Hölder continuous if $f$ is $\varphi_{\alpha}$-continuous.

Let $\psi \in \mathcal{M}$ and $E \subset X$. We consider the family $\Lambda_{\psi}(E)$ of all bounded continuous functions $f$ on $E$ with norm

$$
\|f\|_{\psi, E}=\sup _{x \in E}|f(x)|+\sup _{\substack{x, y \in E \\ x \neq y}} \frac{|f(x)-f(y)|}{\psi(d(x, y))}<\infty .
$$

We define the operator norm

$$
\left\|\mathcal{P}_{\Omega}\right\|_{\psi}=\sup _{\substack{f \in \Lambda_{\psi}(\partial \Omega) \\\|f\|_{\psi, \partial \Omega} \neq 0}} \frac{\left\|\mathcal{P}_{\Omega} f\right\|_{\psi, \Omega}}{\|f\|_{\psi, \partial \Omega}} .
$$

Observe that $\psi$-Hölder continuity of a boundary function $f$ ensures $\psi$-Hölder continuity of $\mathcal{P}_{\Omega} f$ if and only if $\left\|\mathcal{P}_{\Omega}\right\|_{\psi}<\infty$.

Aikawa [2] characterized the family of Euclidean domains $\Omega$ such that $\left\|\mathcal{P}_{\Omega}\right\|_{\psi}<\infty$ for $\psi \in \mathcal{M}$ in context of harmonic functions. We consider the same problem in the context of $p$-harmonic functions in a metric measure space. It is known that there exists $\alpha_{0} \in(0,1]$ such that every $p$-harmonic function in any domain $\Omega$ is locally $\alpha_{0}$-Hölder continuous in $\Omega$ (see [10]). Hence, $\left\|\mathcal{P}_{\Omega}\right\|_{\psi}<\infty$ can hold only for $\psi \in \mathcal{M}$, in some sense, bigger than the function $\varphi_{\alpha_{0}}(t)=t^{\alpha_{0}}$.

Let $\psi, \varphi \in \mathcal{M}$. We say that $\varphi \precsim \psi$ if there are $r_{0}>0$ and $C>0$ such that

$$
\frac{\varphi(s)}{\varphi(r)} \leq C \frac{\psi(s)}{\psi(r)} \quad \text { for } 0<s<r<r_{0} .
$$

Let $\mathcal{M}_{0}$ be the family of all $\psi \in \mathcal{M}$ with $t^{\alpha_{0}} \precsim \psi(t)$. For example, if either $0<\alpha<\alpha_{0}$ and $\beta \in \mathbf{R}$ or $\alpha=0$ and $\beta>0$, then $\psi_{\alpha \beta} \in \mathcal{M}_{0}$. But if $\alpha=\alpha_{0}$ and $\beta<0$, then $\psi_{\alpha_{0} \beta} \notin \mathcal{M}_{0}$. Hence we see that $\mathcal{M}_{0} \varsubsetneqq \mathcal{M}$. Our results will be given for $\psi \in \mathcal{M}_{0}$.

Let $U$ be an open set in $X$ and let $E$ be a Borel set in $\partial U$. We denote by $\omega_{p}(x, E, U)$ the $p$-harmonic measure evaluated at $x$ of $E$ in $U$. Note that the $p$ harmonic measure is not a measure, i.e., the $p$-harmonic measure is not additive. We define two decay properties for $p$-harmonic measures. We say that $\Omega$ enjoys the Local Harmonic Measure Decay property with $\psi$ (abbreviated to the $\operatorname{LHMD}(\psi)$ property) 
if there are positive constants $C_{1}$ and $r_{0}$ depending only $\Omega$ and $\psi$ such that

$$
\omega_{p}(x, \Omega \cap \partial B(a, r), \Omega \cap B(a, r)) \leq C_{1} \frac{\psi(d(x, a))}{\psi(r)} \quad \text { for } x \in \Omega \cap B(a, r),
$$

whenever $\mathrm{a} \in \partial \Omega$ and $0<r<r_{0}$. We say that $\Omega$ enjoys the Global Harmonic Measure Decay property with $\psi$ (abbreviated to the $\operatorname{GHMD}(\psi)$ property) if there are positive constants $C_{2}$ and $r_{0}$ depending only $\Omega$ and $\psi$ such that

$$
\omega_{p}(x, \partial \Omega \backslash B(a, r), \Omega) \leq C_{2} \frac{\psi(d(x, a))}{\psi(r)} \text { for } x \in \Omega \cap B(a, r),
$$

whenever $\mathrm{a} \in \partial \Omega$ and $0<r<r_{0}$. By the comparison principle (see [9, Theorem 7.2]) it is easy to see that (1.1) implies (1.2).

Without loss of generality, we may assume that $\Omega$ is a bounded $p$-regular domain (see [3, Proposition 2.1]). For $a \in \partial \Omega$ we define a test function $\tau_{a, \psi}$ on $\partial \Omega$ by

$$
\tau_{a, \psi}(\xi)=\psi(d(a, \xi)) \quad \text { for } \xi \in \partial \Omega .
$$

Then we have the following theorem.

Theorem 1.2. Let $\psi \in \mathcal{M}_{0}$ and let $\Omega$ be a bounded $p$-regular domain. Consider the following conditions:

(i) $\left\|\mathcal{P}_{\Omega}\right\|_{\psi}<\infty$.

(ii) There is a constant $C$ such that

$$
\mathcal{P}_{\Omega} \tau_{a, \psi}(x) \leq C \psi(d(x, a)) \quad \text { for } x \in \Omega,
$$

whenever $a \in \partial \Omega$.

(iii) $\Omega$ satisfies the $\operatorname{GHMD}(\psi)$ property.

(iv) $\Omega$ satisfies the $\operatorname{LHMD}(\psi)$ property.

Then we have

$$
\text { (i) } \Longleftrightarrow \text { (ii) } \Longrightarrow \text { (iii) } \Longleftarrow \text { (iv) }
$$

The remaining implications in Theorem 1.2 are of interest. Theorem 4.1 in Section 4 will give the equivalence (iii) $\Longleftrightarrow$ (iv) under additional assumptions on $X$ and $\psi \in \mathcal{M}_{0}$. As was observed in [3, Remark 2.4], the implication (iv) $\Longrightarrow$ (i) does not hold. However, we prove that a condition slightly stronger than (iv) implies (i).

Theorem 1.3. Let $\psi, \psi_{1} \in \mathcal{M}_{0}$. Let $\psi_{2}=\psi_{1} / \psi$. Suppose that $\lim _{r \rightarrow 0} \psi_{2}(r)=0$ and there are constants $0<C_{3}<1$ and $r_{0}>0$ such that $\psi_{2}$ is increasing on $\left(0, r_{0}\right)$ and

$$
\sup _{0<\rho<r \leq r_{0}}\left\{\frac{\psi(r)}{\psi(\rho)}: \frac{\psi_{2}(\rho)}{\psi_{2}(r)}=C_{3}\right\}<\infty .
$$

If $\Omega$ satisfies the $\operatorname{LHMD}\left(\psi_{1}\right)$ property, then $\left\|\mathcal{P}_{\Omega}\right\|_{\psi}<\infty$.

Condition (1.3) looks rather complicated. We have a simple condition.

Corollary 1.4. Let $\psi, \psi_{1} \in \mathcal{M}_{0}$. Let $\psi_{2}=\psi_{1} / \psi$. Suppose that there are constants $0<C_{4}<1$ and $r_{0}>0$ such that $\psi$ is increasing on $\left(0, r_{0}\right)$ and

$$
\inf _{0<r \leq r_{0}} \frac{\psi_{2}(r)}{\psi_{2}\left(C_{4} r\right)}>1
$$

If $\Omega$ satisfies the $\operatorname{LHMD}\left(\psi_{1}\right)$ property, then $\left\|\mathcal{P}_{\Omega}\right\|_{\psi}<\infty$. 
Theorem 1.3 and Corollary 1.4 are main results of this paper. They give several corollaries for $\psi_{\alpha \beta}$.

Corollary 1.5. Let $\Omega$ be a bounded p-regular domain. Consider the following conditions:

(i) $0<\alpha<\alpha^{\prime}<\alpha_{0}$ and $\beta, \beta^{\prime} \in \mathbf{R}$.

(ii) $0=\alpha<\alpha^{\prime}<\alpha_{0}$ and $\beta>0, \beta^{\prime} \in \mathbf{R}$.

(iii) $\alpha=\alpha^{\prime}=0$ and $0<\beta<\beta^{\prime}$.

Assume that either (i), (ii), or (iii) holds. If $\Omega$ satisfies the $\operatorname{LHMD}\left(\psi_{\alpha^{\prime} \beta^{\prime}}\right)$ property, then $\left\|\mathcal{P}_{\Omega}\right\|_{\psi_{\alpha \beta}}<\infty$.

We say that $E \subset X$ is uniformly $p$-fat or satisfies the $p$-capacity density condition if there are constants $C>0$ and $r_{0}>0$ such that

$$
\frac{\operatorname{Cap}_{p}(E \cap B(a, r), B(a, 2 r))}{\operatorname{Cap}_{p}(B(a, r), B(a, 2 r))} \geq C,
$$

whenever $a \in E$ and $0<r<r_{0}$. The uniform $p$-fatness of the complement of a domain $\Omega$ is closely related to the condition $\left\|\mathcal{P}_{\Omega}\right\|_{\psi_{\alpha \beta}}<\infty$. For $\alpha>0$ we obtain the following corollary.

Corollary 1.6. Let $\Omega$ be a bounded p-regular domain. If $X \backslash \Omega$ is uniformly $p$-fat, then there is a constant $0<\alpha_{1} \leq \alpha_{0}$ such that $\left\|\mathcal{P}_{\Omega}\right\|_{\psi_{\alpha \beta}}<\infty$ for $0<\alpha<\alpha_{1}$ and $\beta \in \mathbf{R}$. Conversely, if $\left\|\mathcal{P}_{\Omega}\right\|_{\psi_{\alpha \beta}}<\infty$ for some $0<\alpha<\alpha_{0}$ and $\beta \in \mathbf{R}$, then $X \backslash \Omega$ is uniformly $p$-fat, provided that there is a constant $Q \geq p$ such that $X$ is Ahlfors $Q$-regular, i.e.,

$$
C^{-1} r^{Q} \leq \mu(B(x, r)) \leq C r^{Q}
$$

for every $x \in X$ and $r>0$.

Aikawa and Shanmugalingam [3] showed the case $\beta=0$ of Corollary 1.6. For $\alpha=0$ we obtain the following corollary.

Corollary 1.7. If $X \backslash \Omega$ is uniformly $p$-fat, then $\left\|\mathcal{P}_{\Omega}\right\|_{\psi_{0 \beta}}<\infty$ for every $\beta>0$.

The plan of this paper is as follows. In the next section we shall define notions of $p$-harmonicity, $p$-Dirichlet problem, $p$-capacity, and $p$-harmonic measure, and we shall observe some properties for $\mathcal{M}$. In Section 3 we shall show Theorem 1.2. In Section 4 we shall prove that $\Omega$ satisfies the $\operatorname{LHMD}(\psi)$ property if and only if $\Omega$ satisfies the $\operatorname{GHMD}(\psi)$ property under certain additional assumptions. The proof of Theorem 1.3 and Corollary 1.4 will be given in Section 5. Finally, we shall give the proof of Corollaries 1.5, 1.6, and 1.7.

\section{Preliminaries}

In this section we introduce notions of $p$-harmonicity, $p$-Dirichlet problem, $p$ capacity, and $p$-harmonic measure; for details we refer to [3], and we observe some properties for $\mathcal{M}$.

The integral mean of $u$ over a measurable set $E$ is denoted by

$$
\frac{1}{\mu(E)} \int_{E} u d \mu=f_{E} u d \mu=u_{E} .
$$


Definition 2.1. We say that a Borel function $g$ on $X$ is an upper gradient of a real-valued function $u$ on $X$ if

$$
|u(x)-u(y)| \leq \int_{\gamma} g d s
$$

for any $x, y \in X$ and all compact rectifiable curves $\gamma$ joining $x$ and $y$. If (2.1) fails only for a curve family with zero $p$-modulus (see [7, Definition 2.1]), then $g$ is said to be a $p$-weak upper gradient of $u$. We say that $g$ is a minimal $p$-weak upper gradient of $u$ if $g \leq g^{\prime} \mu$-almost everywhere for another $p$-weak upper gradients $g^{\prime}$ of $u$. We denote by $g_{u}$ a minimal $p$-weak upper gradient of $u$.

Definition 2.2. Let $u \in L^{p}(X)$. We define the seminorm

$$
\|u\|_{N^{1, p}(X)}=\|u\|_{L p}+\inf _{g}\|g\|_{L p},
$$

where the infimum is taken over all $p$-weak upper gradients $g$ of $\mathrm{u}$. The Newtonian space on $X$ is the quotient space

$$
N^{1, p}(X)=\left\{u \in L^{p}(X):\|u\|_{N^{1, p}(X)}<\infty\right\} / \sim,
$$

where $u \sim v$ if and only if $\|u-v\|_{N^{1, p}(X)}=0$.

Remark 2.3. The Newtonian space $N^{1, p}(X)$ with the norm $\|\cdot\|_{N^{1, p}(X)}$ is a Banach space. Every function $u \in N^{1, p}(X)$ has the minimal $p$-weak upper gradient $g_{u}$.

Definition 2.4. We say that $X$ admits a $(1, p)$-Poincaré inequality if there are constants $\kappa \geq 1$ and $C \geq 1$ such that for all balls $B(x, r) \subset X$, all measurable functions $u$ on $X$, and all $p$-weak upper gradients $g$ of $u$ we have

$$
f_{B(x, r)}\left|u-u_{B(x, r)}\right| d \mu \leq C r\left(f_{B(x, \kappa r)} g^{p} d \mu\right)^{1 / p} .
$$

A consequence of the $(1, p)$-Poincare inequality is the following $p$-Sobolev inequality (see [10, Lemma 2.1]): if $0<\gamma<1$ and $\mu(\{z \in B(x, R):|u(z)|>0\}) \leq$ $\gamma \mu(B(x, R))$, then there exists a positive constant $C_{\gamma}$ depending only on $\gamma$ such that

$$
\left(f_{B(x, R)}|u|^{p} d \mu\right)^{1 / p} \leq C_{\gamma} R\left(f_{B(x, \kappa R)} g_{u}^{p} d \mu\right)^{1 / p} .
$$

If $X$ admits a $(1, p)$-Poincaré inequality, then $X$ admits a $(1, q)$-Poincaré inequality for every $q \geq p$ by Hölder's inequality. Keith and Zhong [8] showed that if $X$ is proper (that is, closed and bounded subsets of $X$ are compact) and $X$ admits a $(1, p)$-Poincaré inequality, then there exists $q<p$ such that $X$ admits a $(1, q)$ Poincaré inequality. Because $X$ is a complete metric space equipped with a doubling measure, $X$ is proper. Therefore we can use their result.

Definition 2.5. The p-capacity of a subset $E \subset X$ is defined by

$$
\operatorname{Cap}_{p}(E)=\inf _{u}\|u\|_{N^{1, p}(X)}^{p},
$$

where the infimum is taken over all $u \in N^{1, p}(X)$ such that $u \geq 1$ on $E$.

We say that a property holds $p$-quasieverywhere ( $p$-q.e.) if the set of points for which the property fails to hold has $p$-capacity zero. We let

$$
N_{0}^{1, p}(\Omega)=\left\{u \in N^{1, p}(X): u=0 \text { p-q.e. on } X \backslash \Omega\right\} \text {. }
$$


We say that $u \in N_{l o c}^{1, p}(\Omega)$ if for every $x \in \Omega$ there is $r_{x}$ such that $\left.f\right|_{B\left(x, r_{x}\right)} \in$ $N^{1, p}\left(B\left(x, r_{x}\right)\right)$. This is clearly equivalent to saying that $f \in N^{1, p}(V)$ for every relatively compact subset $V$ of $\Omega$. We now introduce the notion of $p$-harmonicity.

Definition 2.6. We call a function $u \in N_{l o c}^{1, p}(\Omega)$ a $p$-harmonic function in $\Omega$ if $u$ is continuous and

$$
\int_{U} g_{u}^{p} d \mu \leq \int_{U} g_{u+\varphi}^{p} d \mu
$$

for all relatively compact subsets $U$ of $\Omega$ and all functions $\varphi \in N_{0}^{1, p}(U)$. A function $u \in N_{\text {loc }}^{1, p}(\Omega)$ is said to be a p-superminimizer in $\Omega$ if $(2.4)$ holds for all relatively compact subsets $U$ of $\Omega$ and all nonnegative functions $\varphi \in N_{0}^{1, p}(U)$. We call a function $u \in N_{l o c}^{1, p}(\Omega)$ a $p$-subminimizer in $\Omega$ if (2.4) holds for all relatively compact subsets $U$ of $\Omega$ and all nonpositive functions $\varphi \in N_{0}^{1, p}(U)$.

Let $u$ and $v$ be $p$-harmonic functions and let $\alpha, \beta \in \mathbf{R}$. Then $\alpha u+\beta$ is $p$-harmonic. But in general $u+v$ is not $p$-harmonic. Kinnunen and Shanmugalingam [10, Theorem 5.2] showed the following local Hölder continuity of $p$-harmonic functions. Here, we denote by $\operatorname{osc}_{E} u$ the oscillation $\sup _{E} u-\inf _{E} u$.

Theorem 2.7. Suppose a function $u$ is $p$-harmonic on $B(x, 2 \kappa R)$. Then there are constants $0<\alpha_{0} \leq 1$ and $C \geq 1$ such that

$$
\underset{B(x, \kappa r)}{\operatorname{Osc}} u \leq C\left(\frac{r}{R}\right)^{\alpha_{0}} \underset{B(x, \kappa R)}{\operatorname{Osc}} u \text { for } 0<r \leq R .
$$

The constants $\alpha_{0}$ and $C$ are independent of $u, x$, and $R$.

Next we define $p$-Dirichlet solutions over $\Omega$. For a function $f \in N^{1, p}(\Omega)$ we denote by $\mathcal{H}_{\Omega} f$ the Dirichlet solution of $f$ over $\Omega$, i.e., $\mathcal{H}_{\Omega} f$ is a function on $\bar{\Omega}$ that is $p$-harmonic in $\Omega$ with $f-\mathcal{H}_{\Omega} f \in N_{0}^{1, p}(\Omega)$. For $E \subset X$ we $\operatorname{denote}$ by $\operatorname{Lip}(E)$ the family of all Lipschitz continuous functions on $E$. For every $f \in \operatorname{Lip}(\partial \Omega)$ there is a function $E f \in \operatorname{Lip}(\bar{\Omega})$ such that $f=E f$ on $\partial \Omega$. Therefore we can define $\mathcal{H}_{\Omega} f$ by the function $\mathcal{H}_{\Omega} E f$; this is independent of the extension $E f$. We say that a lower semicontinuous function $u$ on $\Omega$ is a p-superharmonic function in $\Omega$ if

(i) $-\infty<u \leq \infty$;

(ii) $u$ is not identically $\infty$ in $\Omega$;

(iii) $\mathcal{H}_{\Omega^{\prime}} v \leq u$ in $\Omega^{\prime}$ for every relatively compact subset $\Omega^{\prime}$ of $\Omega$ and all functions $v \in \operatorname{Lip}\left(\partial \Omega^{\prime}\right)$ such that $v \leq u$ on $\partial \Omega^{\prime}$.

If $-u$ is $p$-superharmonic, then we say that $u$ is $p$-subharmonic.

The following comparison principle is very useful in nonlinear potential theory (see [9, Theorem 7.2]).

Theorem 2.8. Let $u$ be a $p$-superharmonic function on $\Omega$ and let $v$ be a $p$ subharmonic function on $\Omega$. If

$$
\limsup _{\Omega \ni x \rightarrow \xi} v(x) \leq \liminf _{\Omega \ni x \rightarrow \xi} u(x)
$$

for every $\xi \in \partial \Omega$, and if both sides of (2.5) are not simultaneously $\infty$ or $-\infty$, then $v \leq u$ in $\Omega$.

Definition 2.9. Let $f$ be a function on $\partial \Omega$. Let $\mathcal{U}_{f}$ be the set of all $p$-superharmonic functions $u$ on $\Omega$ bounded below such that $\liminf _{\Omega \ni x \rightarrow \xi} u(x) \geq f(\xi)$ for each $\xi \in \partial \Omega$. 
The upper Perron solution of $f$ is defined by

$$
\overline{\mathcal{P}}_{\Omega} f(x)=\inf _{u \in \mathcal{U}_{f}} u(x) \quad \text { for } x \in \Omega .
$$

Similarly, we define the lower Perron solution of $f$ by

$$
\underline{\mathcal{P}}_{\Omega} f(x)=\sup _{s \in \mathcal{L}_{f}} s(x) \quad \text { for } x \in \Omega,
$$

where $\mathcal{L}_{f}=-\mathcal{U}_{-f}$ is the set of all $p$-subharmonic functions $s$ on $\Omega$ bounded above such that $\lim \sup _{\Omega \ni x \rightarrow \xi} s(x) \leq f(\xi)$ for each $\xi \in \partial \Omega$. If $\overline{\mathcal{P}}_{\Omega} f=\underline{\mathcal{P}}_{\Omega} f$, then we write $\mathcal{P}_{\Omega} f=\overline{\mathcal{P}}_{\Omega} f$, and we say that $f$ is resolutive. We call $\mathcal{P}_{\Omega} f$ the Perron solution of $f$.

A. Björn, J. Björn and Shanmugalingam [4, Theorem 6.1] showed that if $f \in$ $C(\partial \Omega)$, then $f$ is resolutive. Moreover, if $f \in N^{1, p}(X)$, then $f$ is resolutive and $\mathcal{P}_{\Omega} f=\mathcal{H}_{\Omega} f$, by [4, Theorem 5.1]. We define the $p$-harmonic measure as follows.

Definition 2.10. Let $U$ be an open subset of $X$ and let $E$ be a Borel set in $\partial U$. The $p$-harmonic measure evaluated at $x$ of $E$ in $U$ is defined by

$$
\omega_{p}(x, E, U)=\overline{\mathcal{P}}_{U} \chi_{E}(x) \text { for } x \in U .
$$

The $p$-harmonic measure is not additive because of the non-linear nature of $p$ harmonic functions. Therefore the $p$-harmonic measure is not a measure.

Definition 2.11. Let $E \subset U \subset X$. We define the relative p-capacity of $E$ in $U$ by

$$
\operatorname{Cap}_{p}(E, U)=\inf _{u} \int_{U} g_{u}^{p} d \mu
$$

where the infimum is taken over all $u \in N_{0}^{1, p}(U)$ such that $u \geq 1$ on $E$.

Finally, we observe some properties for $\mathcal{M}$. The following proposition shows an elementary property for $\mathcal{M}$ (see [2, Lemma 2.2]).

Proposition 2.12. Let $\psi \in \mathcal{M}$. If $c>1$ and $0<s \leq t \leq c s$, then $\psi(s) \leq$ $\psi(t) \leq c \psi(s)$.

In Section 1 we have assumed that $\psi \in \mathcal{M}$ is concave. The relevance of concavity of $\psi \in \mathcal{M}$ follows from the following propositions.

Proposition 2.13. Let $\varphi$ be a nondecreasing subadditive function on $(0, \infty)$, i.e., if $t_{1}, t_{2}>0$, then $\varphi\left(t_{1}+t_{2}\right) \leq \varphi\left(t_{1}\right)+\varphi\left(t_{2}\right)$. Suppose that $\lim _{t \rightarrow 0} \varphi(t)=\varphi(0)=0$. Then there is a function $\psi \in \mathcal{M}$ satisfying

$$
\frac{1}{2} \psi(t) \leq \varphi(t) \leq \psi(t) \quad \text { for } t \geq 0
$$

Proposition 2.14. Let $\left(A, d_{A}\right)$ be a geodesic space and let $f$ be a uniformly continuous function on $A$. Then

$$
\varphi(t)=\varphi(f, t)=\sup _{\substack{d_{A}(x, y) \leq t \\ x, y \in A}}|f(x)-f(y)| \quad \text { for } t \geq 0 .
$$

is a subadditive function on $(0, \infty)$.

See [2, Section 5], [5, Chapter $2 \S 6]$, and [11, Section 3] for these accounts. 


\section{Proof of Theorem 1.2}

To prove Theorem 1.2 we recall the following geometric property (see [6, Proposition 4.4]).

Lemma 3.1. The space $X$ is quasiconvex, i.e., there exists a constant $C_{5} \geq 1$ such that every pair of points $x, y \in X$ can be joined by a curve of length at most $C_{5} d(x, y)$. Hence if $x \in E \varsubsetneqq X$, then

$$
\operatorname{dist}(x, X \backslash E) \leq \operatorname{dist}(x, \partial E) \leq C_{5} \operatorname{dist}(x, X \backslash E) .
$$

Proof of Theorem 1.2. Since the $\operatorname{LHMD}(\psi)$ property implies the $\operatorname{GHMD}(\psi)$ property, it is sufficient to show that Condition (ii) implies Condition (iii) and that Condition (i) is equivalent to Condition (ii).

(ii) $\Longrightarrow$ (iii). Suppose (ii) holds. Let $a \in \partial \Omega$ and $r>0$. Then

$$
\psi(r) \chi_{\partial \Omega \backslash B(a, r)}(\xi) \leq \tau_{a, \psi}(\xi) \text { for } \xi \in \partial \Omega .
$$

The comparison principle yields

$$
\psi(r) \omega_{p}(x, \partial \Omega \backslash B(a, r), \Omega) \leq \mathcal{P}_{\Omega} \tau_{a, \psi}(x) \quad \text { for } x \in \Omega .
$$

Hence, (ii) implies that

$$
\psi(r) \omega_{p}(x, \partial \Omega \backslash B(a, r), \Omega) \leq C \psi(d(x, a)) \quad \text { for } x \in \Omega .
$$

Thus (iii) follows.

(i) $\Longrightarrow$ (ii). Suppose $\left\|\mathcal{P}_{\Omega}\right\|_{\psi}<\infty$. Since $\tau_{a, \psi} \in \Lambda_{\psi}(\partial \Omega)$, we have

$$
\left\|\mathcal{P}_{\Omega} \tau_{a, \psi}\right\|_{\psi, \Omega} \leq\left\|\mathcal{P}_{\Omega}\right\|_{\psi}\left\|\tau_{a, \psi}\right\|_{\psi, \partial \Omega}<\infty
$$

By definition

$$
\left|\mathcal{P}_{\Omega} \tau_{a, \psi}(x)-\mathcal{P}_{\Omega} \tau_{a, \psi}(y)\right| \leq\left\|\mathcal{P}_{\Omega} \tau_{a, \psi}\right\|_{\psi, \Omega} \psi(d(x, y)) \quad \text { for } x, y \in \Omega
$$

Letting $y \rightarrow a$, we see that $\mathcal{P}_{\Omega} \tau_{a, \psi}(x) \leq\left\|\mathcal{P}_{\Omega} \tau_{a, \psi}\right\|_{\psi, \Omega} \psi(d(x, a))$. Thus (ii) follows with $C=\left\|\mathcal{P}_{\Omega} \tau_{a, \psi}\right\|_{\psi, \Omega}$.

(ii) $\Longrightarrow$ (i). Suppose (ii) holds. Let $f \in \Lambda_{\psi}(\partial \Omega)$. Since $\left|\mathcal{P}_{\Omega} f\right|$ is bounded by the supremum of $|f|$ over $\partial \Omega$, it is sufficient to show that

$$
\left|\mathcal{P}_{\Omega} f(x)-\mathcal{P}_{\Omega} f(y)\right| \leq C\|f\|_{\psi, \partial \Omega} \psi(d(x, y)) \quad \text { for } x, y \in \Omega .
$$

Let $x, y \in \Omega$. Without loss of generality, we may assume that $\operatorname{dist}(x, X \backslash \Omega) \geq$ $\operatorname{dist}(y, X \backslash \Omega)$. Let $R=\operatorname{dist}(x, X \backslash \Omega) / 2 \kappa$. Since $\partial \Omega$ is compact, we can take $x^{*} \in \partial \Omega$ such that $d\left(x, x^{*}\right)=\operatorname{dist}(x, \partial \Omega)$. Then Lemma 3.1 gives

$$
2 \kappa R \leq d\left(x, x^{*}\right) \leq 2 \kappa C_{5} R \text {. }
$$

Let $f_{0}(\xi)=f(\xi)-f\left(x^{*}\right)$. By definition

$$
\left|f_{0}(\xi)\right| \leq\|f\|_{\psi, \partial \Omega} \tau_{x^{*}, \psi}(\xi) \text { for } \xi \in \partial \Omega .
$$

Hence, by the comparison principle and (ii), we obtain

$$
\left|\mathcal{P}_{\Omega} f_{0}(z)\right| \leq C\|f\|_{\psi, \partial \Omega} \psi\left(d\left(z, x^{*}\right)\right) \quad \text { for } z \in \Omega .
$$

Let us consider two cases.

Case 1: $d(x, y) \leq d\left(x, x^{*}\right) /\left(2 \kappa C_{5}\right)$. Let $r=d(x, y)$. Then $r \leq R$. Since $\mathcal{P}_{\Omega} f_{0}$ is $p$-harmonic, Theorem 2.7 gives

$$
\underset{B(x, \kappa r)}{\operatorname{osc}} \mathcal{P}_{\Omega} f_{0} \leq C\left(\frac{r}{R}\right)^{\alpha_{0}} \underset{B(x, \kappa R)}{\operatorname{osc}} \mathcal{P}_{\Omega} f_{0} .
$$


We obtain from (3.2) that

$$
d\left(z, x^{*}\right) \leq d(x, z)+d\left(x, x^{*}\right) \leq\left(1+2 C_{5}\right) \kappa R \quad \text { for } z \in B(x, \kappa R) .
$$

By Proposition 2.12 we have

$$
\psi\left(d\left(z, x^{*}\right)\right) \leq \psi\left(\left(1+2 C_{5}\right) \kappa R\right) \leq\left(1+2 C_{5}\right) \kappa \psi(R) .
$$

Thus by (3.3) we obtain

$$
\underset{B(x, \kappa R)}{\operatorname{osc}} \mathcal{P}_{\Omega} f_{0} \leq 2 \sup _{B(x, \kappa R)}\left|\mathcal{P}_{\Omega} f_{0}\right| \leq C\|f\|_{\psi, \partial \Omega} \psi(R) .
$$

Hence

$$
\left|\mathcal{P}_{\Omega} f(x)-\mathcal{P}_{\Omega} f(y)\right|=\left|\mathcal{P}_{\Omega} f_{0}(x)-\mathcal{P}_{\Omega} f_{0}(y)\right| \leq C\left(\frac{r}{R}\right)^{\alpha_{0}}\|f\|_{\psi, \partial \Omega} \psi(R) .
$$

Since $\psi \in \mathcal{M}_{0}$, there is a constant $C>0$ such that

$$
\left(\frac{s}{r}\right)^{\alpha_{0}} \leq C \frac{\psi(s)}{\psi(r)} \text { for } 0<s<r<2 \kappa \operatorname{diam}(\Omega) .
$$

Hence by (3.4), we have

$$
\left|\mathcal{P}_{\Omega} f(x)-\mathcal{P}_{\Omega} f(y)\right| \leq C\|f\|_{\psi, \partial \Omega} \psi(d(x, y)) .
$$

Case 2: $d(x, y) \geq d\left(x, x^{*}\right) /\left(2 \kappa C_{5}\right)$. We have

$$
d\left(y, x^{*}\right) \leq d(x, y)+d\left(x, x^{*}\right) \leq\left(1+2 \kappa C_{5}\right) d(x, y) .
$$

It follows from Proposition 2.12 and (3.3) that

$$
\begin{aligned}
\left|\mathcal{P}_{\Omega} f(x)-\mathcal{P}_{\Omega} f(y)\right| & =\left|\mathcal{P}_{\Omega} f_{0}(x)-\mathcal{P}_{\Omega} f_{0}(y)\right| \leq\left|\mathcal{P}_{\Omega} f_{0}(x)\right|+\left|\mathcal{P}_{\Omega} f_{0}(y)\right| \\
& \leq C\|f\|_{\psi, \partial \Omega}\left(\psi\left(d\left(x, x^{*}\right)\right)+\psi\left(d\left(y, x^{*}\right)\right)\right) \\
& \leq C\|f\|_{\psi, \partial \Omega} \psi(d(x, y)) .
\end{aligned}
$$

Combining the above two cases, we obtain (3.1). Thus (i) follows.

\section{Equivalence between $\operatorname{GHMD}(\psi)$ and $\operatorname{LHMD}(\psi)$}

If $\psi=\varphi_{\alpha}$, then the $\operatorname{GHMD}(\psi)$ property and the $\operatorname{LHMD}(\psi)$ property are equivalent for Euclidean domains (see [1]) and for a metric measure space (see [3]). If $\psi \neq \varphi_{\alpha}$, it is not known whether this equivalence holds or not. In this section we show that the equivalence holds under certain additional assumptions.

Let $S(x, r)=\{y \in X: d(x, y)=r\}$ be the sphere with center at $x$ and radius $r$ and let $A(x, r, R)$ be the annulus $B(x, R) \backslash B(x, r)$ with center at $x$ and radii $r$ and $R$. We say that $X$ is linearly locally connected (abbreviated to LLC) if there are constants $C_{6}>1$ and $r_{0}>0$ such that for every $a \in X$ and $0<r<r_{0}$ each pair of points $x, y \in S(a, r)$ can be connected by a curve lying in $A\left(a, r / C_{6}, C_{6} r\right)$.

Theorem 4.1. Let $\Omega$ be a bounded regular domain. Assume that $X$ is $L L C$ and there is a constant $C>0$ such that

$$
\frac{\mu(B(a, r))}{\mu(B(a, R))} \leq C\left(\frac{r}{R}\right)^{p}
$$


whenever a $\in \partial \Omega$ and $0<r \leq R<\operatorname{diam}(X)$. Let $\psi \in \mathcal{M}_{0}$. Suppose that there exist constants $0<C<1$ and $r_{0}>0$ such that

$$
\inf _{0<r<r_{0}} \frac{\psi(r)}{\psi(C r)}>1 .
$$

Then $\Omega$ satisfies the $\operatorname{LHMD}(\psi)$ property if and only if $\Omega$ satisfies the $\operatorname{GHMD}(\psi)$ property.

Theorem 4.1 is new, even for the classical setting, i.e., for harmonic functions in Euclidean domains.

The proof is decomposed mainly into two steps. First, we show that the $\operatorname{GHMD}(\psi)$ property implies that the uniform perfectness of the boundary (Lemma 4.3). Second, with the aid of the uniform perfectness and a chain property, we will complete proof of Theorem 4.1. See [3, Lemmas 5.1 and 5.2] for Hölder continuity.

Definition 4.2. Let $E$ be a subset of $X$. We say that $E$ is uniformly perfect if there are constants $0<C_{7}<1$ and $r_{0}>0$ such that $A\left(x, C_{7} r, r\right) \cap E \neq \emptyset$ for every $x \in E$ and all $0<r<r_{0}$.

Lemma 4.3. Let $\Omega$ be a bounded regular domain. Assume that $X$ is $L L C$ and $\mu$ satisfies (4.1). Let $\psi \in \mathcal{M}_{0}$. Suppose that $\psi$ satisfies (4.2). If $\Omega$ satisfies the $\operatorname{GHMD}(\psi)$ property, then $\partial \Omega$ is uniformly perfect.

For the proof we state the following lemma, which is proved in the same way as [3, Lemma 5.3].

Lemma 4.4. Assume that $\mu$ satisfies (4.1). If $0<2 r \leq R<\operatorname{diam}(\Omega) / 2$, then

$$
\frac{\operatorname{Cap}_{p}(\overline{B(a, r)}, B(a, R))}{\mu(B(a, R))} \leq C\left(\log \frac{R}{r}\right)^{1-p} R^{-p} .
$$

Proof of Lemma 4.3. Let $a \in \partial \Omega$ and $0<\rho_{1}<\rho_{2}<\operatorname{diam}(\Omega) / 2$. Suppose $A\left(a, \rho_{1}, \rho_{2}\right)$ does not intersect $\partial \Omega$. Then it is sufficient to show that the ratio $\rho_{1} / \rho_{2}$ is bounded below by a positive constant $C$ depending only on $\Omega$ and $\psi$.

Without loss of generality, we may assume that $\rho_{1} \leq \rho_{2} /\left(2 C_{6}^{2}\right)$. By the LLC property we see that $A\left(a, C_{6} \rho_{1}, \rho_{2} / C_{6}\right) \subset \Omega$. For simplicity, we let $r=C_{6} \rho_{1}$ and $R=\rho_{2} / C_{6}$. Then

$$
A(a, r, R) \subset \Omega \text {. }
$$

Letting $\rho_{2}$ be larger if necessary, we may assume that $S\left(a, C_{6} R\right)$ has a point $b \in \partial \Omega$. Let $K=\overline{B(a, r)} \backslash \Omega$. Observe from (4.3) that $K=B(a, R) \backslash \Omega$. By Lemma 4.4,

$$
\frac{\operatorname{Cap}_{p}(K, \Omega \cup K)}{\mu(B(a, R))} \leq \frac{\operatorname{Cap}_{p}(\overline{B(a, r)}, B(a, R))}{\mu(B(a, R))} \leq C\left(\log \frac{R}{r}\right)^{1-p} R^{-p} .
$$

Let $u_{K}$ be the $p$-capacitary potential for the condenser $(K, \Omega \cup K)$, i.e., $u_{K}$ is $p$ harmonic on $\Omega, u_{K}=1$ p-q.e. on $K, u_{K}=0$ p-q.e. on $X \backslash(\Omega \cup K)$ and

$$
\operatorname{Cap}_{p}(K, \Omega \cup K)=\int_{X} g_{u_{K}}^{p} d \mu
$$

We prove that $u_{K} \leq 1 / 3 p$-q.e. on $B(b, \beta R)$ for some $0<\beta<1$. Since $r \leq R / 2$ and $A(a, r, R) \cap \partial \Omega=\emptyset$, it follows from the comparison principle and the $\operatorname{GHMD}(\psi)$ 
property that

$$
u_{K}(x) \leq C_{2} \frac{\psi(d(x, b))}{\psi(R / 2)} \quad \text { for } x \in \Omega \cap B(b, R / 2) .
$$

Since $\psi$ satisfies (4.2), there is a constant $0<C_{8}<1$ such that

$$
S=\inf _{0<r<\operatorname{diam}(\Omega) / 2} \frac{\psi(r)}{\psi\left(C_{8} r\right)}>1
$$

Therefore, we have

$$
\frac{\psi\left(C_{8}^{j-1} R / 2\right)}{\psi\left(C_{8}^{j} R / 2\right)} \geq S
$$

for every positive integer $j$. Now multiplying the above inequalities over $j=1,2, \ldots, N$, we get

$$
\frac{\psi(R / 2)}{\psi\left(C_{8}^{N} R / 2\right)} \geq S^{N}
$$

We can find a positive integer $N$ such that

$$
\frac{C_{2}}{S^{N}} \leq \frac{1}{3}
$$

Let $\beta=C_{8}^{N} / 2$. By the monotonicity of $\psi$, if $x \in B(b, \beta R)$, then

$$
\psi(d(x, b)) \leq \psi(\beta R) \leq \psi(R / 2) /\left(3 C_{2}\right) .
$$

Hence, by (4.5) we obtain

$$
u_{K}(x) \leq \frac{1}{3} \quad \text { for } x \in \Omega \cap B(b, \beta R)
$$

Since $u_{K}=0$ p-q.e. on $B(b, R / 2) \backslash \Omega$, we have $u_{K} \leq 1 / 3 p$-q.e. on $B(b, \beta R)$.

Next we prove that $u_{K} \geq 2 / 3 p$-q.e. on $B(a, \beta R)$. It follows from (4.3) and the comparison principle that

$$
u_{K}(x)=1-\omega_{p}(x, \partial \Omega \backslash B(a, R), \Omega) \quad \text { for } x \in \Omega .
$$

By the $\operatorname{GHMD}(\psi)$, we have

$$
\omega_{p}(x, \partial \Omega \backslash B(a, R), \Omega) \leq C_{2} \frac{\psi(d(x, a))}{\psi(R)} \quad \text { for } x \in \Omega \cap B(a, R)
$$

Hence (4.2) implies

$$
u_{K}(x) \geq \frac{2}{3} \quad \text { for } x \in \Omega \cap B(a, \beta R)
$$

Since $u_{K}=1$ p-q.e. on $B(a, \beta R) \backslash \Omega \subset B(a, R) \backslash \Omega$, we obtain $u_{K} \geq 2 / 3$ p-q.e. on $B(a, \beta R)$.

Let $v=\max \left\{u_{K}, 1 / 3\right\}-1 / 3 \geq 0$. Then

$$
\frac{\mu\left(\left\{x \in B\left(a, 2 C_{6} R\right): v(x)=0\right\}\right)}{\mu\left(B\left(a, 2 C_{6} R\right)\right)} \geq \frac{\mu(B(b, \beta R))}{\mu\left(B\left(a, 2 C_{6} R\right)\right)} \geq \gamma
$$

where $\gamma>0$ depends only on $\beta$. Hence the $p$-Sobolev inequality (2.3) and the doubling property of $\mu$ imply

$$
\left(\int_{B\left(a, 2 C_{6} R\right)} v^{p} d \mu\right)^{1 / p} \leq C R\left(\int_{B\left(a, 2 \kappa C_{6}\right)} g_{v}^{p} d \mu\right)^{1 / p} .
$$


By the doubling property of $\mu$ we have

$$
\int_{B\left(a, 2 C_{6} R\right)} v^{p} d \mu \geq \int_{B(a, \beta R)}(1 / 3)^{p} d \mu \geq C \mu(B(a, R)) .
$$

Hence, we obtain

$$
\begin{aligned}
\operatorname{Cap}_{p}(K, \Omega \cup K) & =\int_{X} g_{u_{K}}^{p} d \mu \geq \int_{B\left(a, 2 \kappa C_{6} R\right)} g_{v}^{p} d \mu \\
& \geq C R^{-p} \int_{B\left(a, 2 C_{6} R\right)} v^{p} d \mu \geq C R^{-p} \mu(B(a, R)) .
\end{aligned}
$$

This, together with (4.4), implies that $r / R$ is bounded below and therefore so is $\rho_{1} / \rho_{2}$. Thus the lemma is proved.

To prove Theorem 4.1 we state two lemmas in [3].

Lemma 4.5. Let $0<R<\operatorname{diam}(\Omega) / 6 \kappa$ and let $u$ be a $p$-subminimizer on $B(z, 2 \kappa R)$. Suppose $0 \leq u \leq 1$ on $B(z, 2 \kappa R)$ and

$$
\frac{\mu(\{x \in B(z, R): u(x)>1-s\})}{\mu(B(z, R))} \leq \gamma<1
$$

for some $0<s<1$. Then there exists a constant $t>0$ such that

$$
u \geq 1-t \quad \text { on } B(z \cdot R / 2) \text {. }
$$

The constant $t$ is independent of $u, z$, and $R$.

Lemma 4.6. Let $0<R<\operatorname{diam}(\Omega) / 6 \kappa$. Let $B\left(z_{1}, R / 2\right) \cap B\left(z_{2}, R / 2\right) \neq \emptyset$. Suppose $u$ is a $p$-subminimizer on $B\left(z_{2}, 2 \kappa R\right)$ with $0 \leq u \leq 1$ in $B\left(z_{2}, 2 \kappa R\right)$. If $u \leq$ $1-\varepsilon_{1}$ on $B\left(z_{1}, R / 2\right)$ for some $\varepsilon_{1}>0$, then there is a positive constant $\varepsilon_{2}=\varepsilon_{2}\left(\varepsilon_{1}\right)<1$ such that $u \leq 1-\varepsilon_{2}$ on $B\left(z_{2}, R / 2\right)$.

Proof of Theorem 4.1. It is sufficient to show that if $\Omega$ satisfies the $\operatorname{GHMD}(\psi)$ property, then $\Omega$ satisfies the $\operatorname{LHMD}(\psi)$ property. Since $\Omega$ is uniformly perfect by Lemma 4.3, there are constants $0<C_{7}<1$ and $r_{0}>0$ such that $A\left(x, C_{7} r, r\right) \cap \partial \Omega \neq \emptyset$ for every $x \in \partial \Omega$ and all $0<r<r_{0}$. Let $a \in \partial \Omega$ and $0<r<r_{0}$. Then we can find $\rho$ such that $S(a, \rho) \cap \partial \Omega \neq \emptyset$ and $C_{7} r \leq \rho<r$.

Let $c$ be a small positive number to be determined later. By the LLC property and the doubling property of $\mu$ we can find finitely many points $z_{1}, \ldots, z_{N} \in$ $A\left(a, \rho / C_{6}, C_{6} \rho\right)$ such that the union $\cup_{j=1}^{N} B\left(z_{j}, c r\right)$ is a covering of $S(a, \rho)$ that forms a chain, that is, for every $k, l \in\{1, \ldots, N\}$ there is a subcollection of balls $B_{j_{1}}, \ldots, B_{j_{m}}$ such that $B_{k}=B_{j_{1}}, B_{l}=B_{j_{m}}$ and $B_{j_{i}} \cap B_{j_{i+1}} \neq \emptyset$ for $i \in\{1, \ldots, m-1\}$. Observe that

$$
\bigcup_{j=1}^{N} B\left(z_{j}, 4 \kappa c r\right) \subset A\left(a, \frac{\rho}{C_{6}}-4 \kappa c r, C_{6} \rho+4 \kappa c r\right) \subset A\left(a,\left(\frac{C_{7}}{C_{6}}-4 \kappa c\right) r,\left(C_{6}+4 \kappa c\right) r\right) .
$$

Let $c>0$ be small enough so that $4 \kappa c \leq C_{7} /\left(2 C_{6}\right)$. Let $\eta=C_{7} /\left(2 C_{6}\right)$. Consider

$$
u= \begin{cases}\omega_{p}(\partial \Omega \cap B(a, \eta r), \Omega) & \text { on } \Omega, \\ 0 & \text { on } X \backslash \Omega .\end{cases}
$$

Then $0 \leq u \leq 1$ on $X$ and $u$ is a $p$-subminimizer in $X \backslash \overline{B(a, \eta r)} \supset \cup_{j=1}^{N} B\left(z_{j}, 4 \kappa c r\right)$. Fix $z^{*} \in \partial \Omega \cap S(a, \rho)$. Without loss of generality, we may assume that $z^{*} \in B\left(z_{1}, c r\right)$. 
Since

$$
B\left(z^{*},(4 \kappa-1) c r\right) \subset B\left(z_{1}, 4 \kappa c r\right) \subset X \backslash \overline{B(a, \eta r)}
$$

it follows from the comparison principle that

$$
u(x) \leq \omega_{p}\left(x, \partial \Omega \backslash B\left(z^{*},(4 \kappa-1) c r\right), \Omega\right) \text { for } x \in \Omega .
$$

Since $\Omega$ satisfies the $\operatorname{GHMD}(\psi)$ property and $\psi$ satisfies (4.2), we obtain

$$
u(x) \leq \frac{1}{2} \quad \text { for } x \in B\left(z^{*}, \beta r\right) \cap \Omega
$$

for some $\beta>0$ independent of $a$ and $r$. Since $u=0$ on $X \backslash \Omega$, we have $u \leq 1 / 2$ on $B\left(z^{*}, \beta r\right)$. Hence Lemma 4.5 with $R=2 c r$ yields that $u \leq 1-\varepsilon_{1}$ on $B\left(z_{1}, c r\right)$ for some $\varepsilon_{1}>0$ independent of $a$ and $r$. Since $\cup_{j=1}^{N} B\left(z_{j}, c r\right)$ is a chain, we find some ball, say $B\left(z_{2}, c r\right)$, intersecting $B\left(z_{1}, c r\right)$. Then by Lemma 4.6 we have $u \leq 1-\varepsilon_{2}$ on $B\left(z_{2}, c r\right)$ for some $\varepsilon_{2}>0$. We may repeat this argument finitely many times until, by the finiteness of the cover and its chain property, we eventually obtain $u \leq 1-\varepsilon_{0}$ on $\cup_{j=1}^{N} B\left(z_{j}, c r\right)$ for some $\varepsilon_{0}>0$ that is independent of $a$ and $r$. In particular, $u \leq 1-\varepsilon_{0}$ on $S(a, \rho)$. Since

$$
\omega_{p}(\partial \Omega \cap B(a, \eta r), \Omega)=1-\omega_{p}(\partial \Omega \backslash B(a, \eta r), \Omega) \text { on } \Omega,
$$

it follows that $\omega_{p}(\partial \Omega \backslash B(a, \eta r), \Omega) \geq \varepsilon_{0}$ on $\Omega \cap S(a, \rho)$. By the comparison principle we have

$$
\frac{1}{\varepsilon_{0}} \omega_{p}(\partial \Omega \backslash B(a, \eta r), \Omega) \geq \omega_{p}(\Omega \cap \partial B(a, \rho), \Omega \cap B(a, \rho)) \quad \text { on } \Omega \cap B(a, \rho) .
$$

Hence the $\operatorname{GHMD}(\psi)$ property and Proposition 2.12 yield

$$
\begin{aligned}
\omega_{p}(x, \Omega \cap \partial B(a, r), \Omega \cap B(a, r)) & \leq \omega_{p}(x, \Omega \cap \partial B(a, \rho), \Omega \cap B(a, \rho)) \\
& \leq \frac{C_{2}}{\varepsilon_{0}} \frac{\psi(d(x, a))}{\psi(\eta r)} \leq \frac{C_{2}}{\varepsilon_{0} \eta} \frac{\psi(d(x, a))}{\psi(r)}
\end{aligned}
$$

for all $x \in \Omega \cap B(a, \rho)$. Because $\rho \geq C_{7} r$, we obtain $d(x, a) \geq C_{7} r$ for all $x \in$ $\Omega \cap B(a, r) \backslash B(a, \rho)$. Proposition 2.12 yields

$$
\omega_{p}(x, \Omega \cap \partial B(a, r), \Omega \cap B(a, r)) \leq 1 \leq \frac{\psi(d(x, a))}{\psi\left(C_{7} r\right)} \leq \frac{1}{C_{7}} \frac{\psi(d(x, a))}{\psi(r)}
$$

for all $x \in \Omega \cap B(a, r) \backslash B(a, \rho)$. Thus $\Omega$ satisfies the $\operatorname{LHMD}(\psi)$ property.

Remark 4.7. We say that $X$ is Ahlfors $Q$-regular if there exists a positive constant $C$ such that

$$
C^{-1} r^{Q} \leq \mu(B(x, r)) \leq C r^{Q} \text { for every } B(x, r) .
$$

If $X$ is Ahlfors $Q$-regular with $Q \geq p$, then $\mu$ satisfies (4.1). Moreover if $X$ supports a $(1, p)$-Poincaré inequality and $X$ is Ahlfors $Q$-regular with $Q \geq p$, then $X$ is LLC (see [6, Proposition 4.5]). Therefore, if $X$ is Ahlfors $Q$-regular with $Q \geq p$ and $\psi \in \mathcal{M}_{0}$ satisfies (4.2), then $\Omega$ satisfies the $\operatorname{LHMD}(\psi)$ property if and only if $\Omega$ satisfies the $\operatorname{GHMD}(\psi)$ property.

Remark 4.8. Let $\psi=\psi_{\alpha \beta}$. If $\alpha>0$, then $\psi_{\alpha \beta}$ satisfies (4.2). Therefore if $X$ is Ahlfors $Q$-regular with $Q \geq p$, then the $\operatorname{LHMD}\left(\psi_{\alpha \beta}\right)$ property and the $\operatorname{GHMD}\left(\psi_{\alpha \beta}\right)$ property are equivalent. On the other hand, $\psi_{0 \beta}$ does not satisfy $(4.2)$, and we do not know whether the equivalence holds or not. 


\section{Proof of Theorem 1.3 and Corollary 1.4}

In this section we give the proof of Theorem 1.3 and Corollary 1.4.

Proof of Theorem 1.3. Let $a \in \partial \Omega$ and $u=\mathcal{P}_{\Omega} \tau_{a, \psi}$. We will show (ii) in Theorem 1.2 holds, i.e., $u(x) \leq C \psi(d(x, a))$. For $\rho>0$ we define a function $f(\rho)$ by

$$
f(\rho)=\sup _{\Omega \cap S(a, \rho)} u(x) .
$$

It is sufficient to show that

$$
f(\rho) \leq C \psi(\rho)
$$

for small $\rho>0$. Let $0<\rho<r<\operatorname{diam}(\Omega)$. By definition of $\tau_{a, \psi}$ we see that $u \leq \psi(r)+f(r) \chi_{\Omega \cap \partial B(a, r)}$ on $\partial(\Omega \cap B(a, r))$. The comparison principle yields

$$
u(x) \leq \psi(r)+f(r) \omega_{p}(x, \Omega \cap \partial B(a, r), \Omega \cap B(a, r))
$$

for all $x \in \Omega \cap B(a, r)$. Hence, the $\operatorname{LHMD}\left(\psi_{1}\right)$ property implies

$$
f(\rho) \leq \psi(r)+C_{1} f(r) \frac{\psi_{1}(\rho)}{\psi_{1}(r)}=\psi(r)+C_{1} f(r) \frac{\psi(\rho)}{\psi(r)} \frac{\psi_{2}(\rho)}{\psi_{2}(r)} .
$$

Without loss of generality, we assume that $r_{0}<\operatorname{diam}(\Omega)$. We can find a positive integer $N$ such that $C_{3}^{N} \leq 1 /\left(2 C_{1}\right)$. By (1.3) we have

$$
M=\sup _{0<\rho<r \leq r_{0}}\left\{\frac{\psi(r)}{\psi(\rho)}: \frac{\psi_{2}(\rho)}{\psi_{2}(r)}=C_{3}^{N}\right\}<\infty
$$

We can find the number $0<r_{0}^{\prime}<r_{0}$ such that

$$
\frac{\psi_{2}\left(r_{0}^{\prime}\right)}{\psi_{2}\left(r_{0}\right)}=C_{3}^{N}
$$

Let $0<r<r_{0}^{\prime}$. Then by (5.3) we can find a sequence $\left\{\rho_{j}\right\}_{j=1}^{n}$ such that $r=\rho_{0}<$ $\rho_{1}<\ldots<\rho_{n-1}<r_{0}^{\prime} \leq \rho_{n}<r_{0}$,

$$
\frac{\psi_{2}\left(\rho_{j}\right)}{\psi_{2}\left(\rho_{j+1}\right)}=C_{3}^{N} \leq \frac{1}{2 C_{1}} \quad \text { for } j=0,1, \ldots, n-1
$$

and

$$
\frac{\psi\left(\rho_{j+1}\right)}{\psi\left(\rho_{j}\right)} \leq M \quad \text { for } j=0,1, \ldots, n-1
$$

Hence, by (5.2) we obtain

$$
f\left(\rho_{j}\right) \leq \psi\left(\rho_{j+1}\right)+\frac{1}{2} f\left(\rho_{j+1}\right) \frac{\psi\left(\rho_{j}\right)}{\psi\left(\rho_{j+1}\right)} \quad \text { for } j=0,1, \ldots, n-1
$$


These inequalities imply that

$$
\begin{aligned}
f(r)=f\left(\rho_{0}\right) & \leq \psi\left(\rho_{1}\right)+\psi\left(\rho_{0}\right) \sum_{j=1}^{n-1} \frac{1}{2^{j}} \frac{\psi\left(\rho_{j+1}\right)}{\psi\left(\rho_{j}\right)}+\frac{1}{2^{n}} f\left(\rho_{n}\right) \frac{\psi\left(\rho_{0}\right)}{\psi\left(\rho_{n}\right)} \\
& \leq M \psi\left(\rho_{0}\right)+M \psi\left(\rho_{0}\right) \sum_{j=1}^{n-1} \frac{1}{2^{j}}+f\left(\rho_{n}\right) \frac{\psi\left(\rho_{0}\right)}{\psi\left(\rho_{n}\right)} \\
& \leq M \psi\left(\rho_{0}\right)+M \psi\left(\rho_{0}\right)+f\left(\rho_{n}\right) \frac{\psi\left(\rho_{0}\right)}{\psi\left(\rho_{n}\right)} \\
& \leq\left(2 M+\frac{\psi(\operatorname{diam}(\Omega))}{\psi\left(r_{0}^{\prime}\right)}\right) \psi(r),
\end{aligned}
$$

where $f \leq \psi(\operatorname{diam}(\Omega))$ and $r_{0}^{\prime} \leq \rho_{n}$ are used in the last inequality. Thus (5.1) follows, and so (ii) in Theorem 1.2 holds. Hence $\left\|\mathcal{P}_{\Omega}\right\|_{\psi}<\infty$ by Theorem 1.2.

Proof of Corollary 1.4. Let us prove (1.3) with

$$
C_{3}=\sup _{0<r \leq r_{0}} \frac{\psi_{2}\left(C_{4} r\right)}{\psi_{2}(r)}<1 .
$$

Fix $0<r \leq r_{0}$. Then

$$
\frac{\psi_{2}\left(C_{4} r\right)}{\psi_{2}(r)} \leq C_{3}
$$

By the monotonicity of $\psi_{2}$ we can find a number $\rho$ such that $C_{4} r \leq \rho<r$ and

$$
\frac{\psi_{2}(\rho)}{\psi_{2}(r)}=C_{3}
$$

Proposition 2.12 yields that

$$
\frac{\psi(r)}{\psi(\rho)} \leq \frac{\psi(r)}{\psi\left(C_{4} r\right)} \leq \frac{1}{C_{4}}
$$

Hence we have

$$
\sup _{0<\rho<r \leq r_{0}}\left\{\frac{\psi(r)}{\psi(\rho)}: \frac{\psi_{2}(\rho)}{\psi_{2}(r)}=C_{3}\right\} \leq \frac{1}{C_{4}}<\infty .
$$

Next we prove that $\lim _{r \rightarrow 0} \psi_{2}(r)=0$. By the monotonicity of $\psi_{2}$ the limit of $\psi_{2}(r)$ exists, as $r \rightarrow 0$. If $\lim _{r \rightarrow 0} \psi_{2}(r) \neq 0$, then we would have

$$
\lim _{r \rightarrow 0} \frac{\psi_{2}(r)}{\psi_{2}\left(C_{4} r\right)}=1
$$

This would contradict (1.4). Hence $\lim _{r \rightarrow 0} \psi_{2}(r)=0$. Since the assumptions of Theorem 1.3 are satisfied, it follows that $\left\|\mathcal{P}_{\Omega}\right\|_{\psi}<\infty$.

\section{Proof of Corollaries 1.5, 1.6 and 1.7}

In this section we prove Corollaries 1.5, 1.6, and 1.7.

Proof of Corollary 1.5. We divide the proof into the following two cases.

Case 1: (i) or (ii) holds. Let $\psi=\psi_{\alpha \beta}, \psi_{1}=\psi_{\alpha^{\prime} \beta^{\prime}}$, and $\psi_{2}=\psi_{1} / \psi$. Let $r_{0}$ be a small positive number. Then

$$
\psi_{2}(r)=r^{\alpha^{\prime}-\alpha}(-\log r)^{-\beta^{\prime}+\beta} \text { for } 0<r \leq r_{0} .
$$


Hence $\psi_{2}$ is increasing on $\left(0, r_{0}\right)$, and for some constant $C_{4} \in(0,1)$

$$
\inf _{0<r \leq r_{0}} \frac{\psi_{2}(r)}{\psi_{2}\left(C_{4} r\right)}>1
$$

Since the assumptions of Corollary 1.4 are satisfied, we have $\left\|\mathcal{P}_{\Omega}\right\|_{\psi_{\alpha \beta}}<\infty$.

Case 2: (iii) holds. Let $\psi=\psi_{0 \beta}, \psi_{1}=\psi_{0 \beta^{\prime}}$, and $\psi_{2}=\psi_{1} / \psi$. Let $r_{0}$ be a small positive number. Then

$$
\psi_{2}(r)=(\log r)^{-\beta^{\prime}+\beta} \quad \text { for } 0<r \leq r_{0} .
$$

Hence $\lim _{r \rightarrow 0} \psi_{2}(r)=0$ and $\psi_{2}$ is increasing on $\left(0, r_{0}\right)$. Fix a constant $0<\eta<1$ and $0<r \leq r_{0}$. Let $\lambda=\eta^{1 /\left(\beta-\beta^{\prime}\right)}$ and $\rho=r^{\lambda}$. Then we have

$$
\frac{\psi_{2}(\rho)}{\psi_{2}(r)}=\lambda^{-\beta^{\prime}+\beta}=\eta
$$

and

Hence

$$
\frac{\psi(r)}{\psi(\rho)}=\lambda^{\beta}
$$

$$
\sup _{0<\rho<r \leq r_{0}}\left\{\frac{\psi(r)}{\psi(\rho)}: \frac{\psi_{2}(\rho)}{\psi_{2}(r)}=\eta\right\}=\lambda^{\beta}<\infty .
$$

Thus it follows from Theorem 1.3 that $\left\|\mathcal{P}_{\Omega}\right\|_{\psi_{0 \beta}}<\infty$.

To prove Corollaries 1.6 and 1.7 we observe the following lemma (see [3, Lemma 6.1]).

Lemma 6.1. A domain $\Omega$ satisfies the $\operatorname{LHMD}\left(\varphi_{\alpha_{2}}\right)$ property for some $\alpha_{2}>0$ if and only if $X \backslash \Omega$ is uniformly $p$-fat.

Proof of Corollary 1.6. First suppose that $X \backslash \Omega$ is uniformly $p$-fat. It follows from Lemma 6.1 that there is a constant $\alpha_{2}>0$ such that $\Omega$ satisfies the $\operatorname{LHMD}\left(\varphi_{\alpha_{2}}\right)$ property. Let $\alpha_{1}=\min \left\{\alpha_{0}, \alpha_{2}\right\}$. Then $\Omega$ satisfies the $\operatorname{LHMD}\left(\varphi_{\alpha_{1}}\right)$ property. Corollary 1.5 yields that $\left\|\mathcal{P}_{\Omega}\right\|_{\psi_{\alpha \beta}}<\infty$ for $0<\alpha<\alpha_{1}$ and $\beta \in \mathbf{R}$.

Conversely, suppose that $\left\|\mathcal{P}_{\Omega}\right\|_{\psi_{\alpha \beta}}<\infty$ for some $0<\alpha<\alpha_{0}$ and $\beta \in \mathbf{R}$. Assume that $X$ is Ahlfors $Q$-regular with $Q \geq p$. By Theorem $1.2 \Omega$ satisfies the $\operatorname{GHMD}\left(\psi_{\alpha \beta}\right)$ property. It follows from Remark 4.8 that $\Omega$ satisfies the $\operatorname{LHMD}\left(\psi_{\alpha \beta}\right)$ property. Let $0<\alpha^{\prime}<\alpha$. By Corollary 1.5 we obtain that $\left\|\mathcal{P}_{\Omega}\right\|_{\varphi_{\alpha^{\prime}}}<\infty$. Theorem 1.2 and Theorem 4.1 imply that $\Omega$ satisfies the $\operatorname{LHMD}\left(\varphi_{\alpha^{\prime}}\right)$ property. Lemma 6.1 yields that $X \backslash \Omega$ is uniformly $p$-fat.

Proof of Corollary 1.7. Suppose that $X \backslash \Omega$ is uniformly $p$-fat. It follows from Lemma 6.1 that there is a constant $\alpha_{2}>0$ such that $\Omega$ satisfies the $\operatorname{LHMD}\left(\varphi_{\alpha_{2}}\right)$ property. Let $\alpha_{1}=\min \left\{\alpha_{0}, \alpha_{2}\right\}$. Then $\Omega$ satisfies the $\operatorname{LHMD}\left(\varphi_{\alpha_{1}}\right)$ property. Corollary 1.5 yields that $\left\|\mathcal{P}_{\Omega}\right\|_{\psi_{0 \beta}}<\infty$ for every $\beta \in \mathbf{R}$.

Acknowledgements. The author is grateful to the referee for many valuable comments. 


\section{References}

[1] Aikawa, H.: Hölder continuity of the Dirichlet solution for a general domain. - Bull. Lond. Math. Soc. 34:6, 2002, 691-702.

[2] Aikawa, H.: Modulus of continuity of the Dirichlet solutions. - Bull. Lond. Math. Soc. 42:5, $2010,857-867$.

[3] Aikawa, H, and N. Shanmugalingam: Hölder estimates of p-harmonic extension operators. - J. Differential Equations 220:1, 2006, 18-45.

[4] BJöRn, A., J. BJöRn, and N. Shanmugalingam: The Perron method for $p$-harmonic functions in metric spaces. - J. Differential Equations 195:2 2003, 398-429.

[5] DeVore, R. A., and G. G. Lorentz: Constructive approximation. - Grundlehren Math. Wiss. 303, Springer-Verlag, Berlin, 1993.

[6] HajŁasz, P., and P. Koskela: Sobolev met Poincaré. - Mem. Amer. Math. Soc. 145:688, 2000.

[7] Heinonen, J., and P. Koskela: Quasiconformal maps in metric spaces with controlled geometry. - Acta Math. 181:1, 1998, 1-61.

[8] Keith, S., and X. Zhong: The Poincaré inequality is an open ended condition. - Ann. of Math. (2) 167:2, 2008, 575-599.

[9] Kinnunen, J., and O. Martio: Nonlinear potential theory on metric spaces. - Illinois J. Math. 46:3, 2002, 857-883.

[10] Kinnunen, J., and N. Shanmugalingam: Regularity of quasi-minimizers on metric spaces. - Manuscripta Math. 105:3, 2001, 401-423.

[11] Kuroda, S. T.: Diagonalization modulo norm ideals; spectral method and modulus of continuity. - In: Spectral and scattering theory and related topics, RIMS Kôkyûroku Bessatsu, B16, Res. Inst. Math. Sci. (RIMS), Kyoto, 2010, 101-126.

Received 29 April 2011 